\title{
White light-emitting diodes for optical stimulation of aluminium oxide in OSL dosimetry
}

\author{
A.I. Surdo ${ }^{\mathrm{a}, \mathrm{b}, *}$, I.I. Milman ${ }^{\mathrm{b}}$, M.I. Vlasov ${ }^{\mathrm{a}}$ \\ ${ }^{a}$ Radiation Laboratory, Institute of Industrial Ecology, UB RAS, 20 Sofia Kovalevskay Str., 620990 Ekaterinburg, Russia \\ ${ }^{\mathrm{b}}$ Department of Experimental Physics, Ural Federal University, Ekaterinburg, Russia
}

\section{H I G H L I G H T S}

- Using a white LED increases by more than 2 times the OSL output.

- The use of white LEDs reduces by at least 5 times the decay time.

- Special filling of deep traps at $670 \mathrm{~K}$ is not less than 10 times increase OSL and TL output.

\section{A R T I C L E I N F O}

\section{Article history:}

Received 2 April 2012

Received in revised form

6 May 2013

Accepted 13 May 2013

\section{Keywords:}

Anion-defective corundum

Sources for optical stimulation

Optically stimulated luminescence

Thermoluminescence

Deep traps

\begin{abstract}
A B S T R A C T
The results of comparative investigations into the optically stimulated luminescence (OSL) of aniondefective corundum excited by radiation of blue and white light-emitting diodes (LEDs) are reported. The continuous-wave OSL (CW-OSL) measurements showed that the white LED (compared to the blue LED) and the chosen geometry allow the amplitude of the OSL response to increase by a factor of more than 40 and the dosimetric information readout time to decrease by a factor of 20 .

The effect of deep traps on the behavior of the CW-OSL curves of anion-defective corundum was studied. It was found that the filling of deep traps essentially increases the TL yield with a dosimetric peak at $450 \mathrm{~K}$ and the CW-OSL-yield under stimulation with blue and white LEDs. It was also found that this filling causes a considerable change in the shape of the OSL curve and the time parameters of the decay.
\end{abstract}

(c) 2013 Elsevier Ltd. All rights reserved.

\section{Introduction}

In recent years, optically stimulated luminescence (OSL) has become an increasingly popular method for the detection of ionizing radiation in scientific, technical, medical, and space applications. The physical and hardware aspects of OSL are under active development at leading dosimetric laboratories worldwide (Hashimoto et al., 2002; Bøtter-Jensen et al., 2003; Miller et al., 2006; Yukihara and McKeever, 2011; Denis et al., 2011; Surdo et al., 2013). In the modern practice of OSL dosimetry, detectors based on anion-defective crystals of $\alpha-\mathrm{Al}_{2} \mathrm{O}_{3}$ have gained the widest acceptance. These detectors were designed for use in thermoluminescence (TL) dosimetry and are commercially labeled as TLD500 or $\alpha-\mathrm{Al}_{2} \mathrm{O}_{3}: \mathrm{C}$. However, because of their unique properties, they have proven highly acceptable in OSL applications.

\footnotetext{
* Corresponding author. Radiation Laboratory, Institute of Industrial Ecology, UB RAS, 20 Sofia Kovalevskay Str., 620990 Ekaterinburg, Russia. Tel.: +7 343 3754711; fax: +7 3433754711 .

E-mail address: Surdo@ecko.uran.ru (A.I. Surdo).
}

The OSL method is based on the optically stimulated release of charge carriers from trapping levels filled under ionizing radiation and on the registration of the luminescence caused by the recombination of released carriers on emission centers. The OSL yield is proportional to the absorbed radiation dose and depends on the intensity and length of the stimulating light wave. Lasers and lightemitting diodes (LEDs) are used for stimulation. These stimulation sources are compact and provide the required intensity of the light flux. The OSL signal is separated from the exciting radiation in selective optical filters or by timing with the use of a pulsed stimulation when the OSL signal is registered in time intervals between the exciting light pulses (McKeever and Akselerod, 1999). When determining the OSL stimulation method, preference is given to LEDs with wavelengths of 470, 530, and $890 \mathrm{~nm}$ (Yukihara and McKeever, 2011). A drawback of the aforementioned methods is that the LEDs excite OSL in a narrow spectral range $(\sim 20 \mathrm{~nm})$ and only at a fixed wavelength, which is determined by the LED type. In this case, a well-known dependence of the OSL yield on the stimulating light wavelength in the detectors based on the aniondefective $\alpha-\mathrm{Al}_{2} \mathrm{O}_{3}$ crystals (Bøtter-Jensen et al., 2003) has not 
been considered to its full extent. A maximum OSL yield is achieved upon stimulation by optical radiation with a wavelength of approximately $470 \mathrm{~nm}$. As the wavelength increases, the OSL yield decreases smoothly and remains measurable up to the infrared wavelength range.

The shape of the spectral dependence of the OSL yield is governed by the energy distribution of optically active traps of charge carriers in the forbidden gap of the $\alpha-\mathrm{Al}_{2} \mathrm{O}_{3}$ crystal. The optical depth of these traps ranges between $1.8 \mathrm{eV}(700 \mathrm{~nm})$ and $3.1 \mathrm{eV}$ $(400 \mathrm{~nm})$ (Springis et al., 1995; Walker et al., 1996; Surdo and Kortov, 2004). If the energy of the photons of the stimulating light does not coincide with the optical depth of a trap, the depletion requires a longer stimulation time and stimulating light of higher intensity.

One more drawback of the known methods is that the stimulating radiation strikes the detector surface that faces the photoreceiver at an incidence angle of $50-60^{\circ}$, so the detector has to be installed at least $40-50 \mathrm{~mm}$ away from the entrance window of the photoreceiver (Bøtter-Jensen et al., 2003). The value of the OSL signal is directly related to the stimulating light intensity and affects the sensitivity of the entire system. The loss in the intensity of the stimulating radiation due to an oblique incidence of the light flux and the reflection of some flux from the detector surface is compensated by increasing the radiation power. For this purpose, several tens of LEDs are used in the stimulation system (Miller et al., 2006).

It is also known that the TL yield in the vicinity of the main dosimetric peak at $450 \mathrm{~K}$ strongly depends on the extent of the filling of deep traps depleted at $T>600 \mathrm{~K}$ in the anion-defective corundum crystals (Mil'man et al., 2008; Solov'ev et al., 2012). The similar data for the OSL yield are absent in the literature.

Therefore, the goal of this work is to study the possibility of making the OSL registration more efficient through the expansion of the spectral range of the stimulation source, the adjustment of its position relative to the detector, and the extent of the filling of deep traps.

\section{Experimental details}

The samples under study are nominally pure anion-defective $\alpha$ $\mathrm{Al}_{2} \mathrm{O}_{3}$ single crystals of a cylindrical form that are $5 \mathrm{~mm}$ in diameter and $1 \mathrm{~mm}$ in thickness (standard detectors of the TLD-500 type). They were chosen due to their high OSL- and TL-yields.

The CW-OSL decay curves and the TL were measured using a home-built automatic reader. The OSL and TL were measured after irradiation with a ${ }^{90} \mathrm{Sr} /{ }^{90} \mathrm{Y}$ beta source.

Fig. 1 shows the schematic diagram of the used OSL unit. To reduce the loss of the stimulating light, the sample (2) was placed between the optical stimulation source (1) and a separation optical

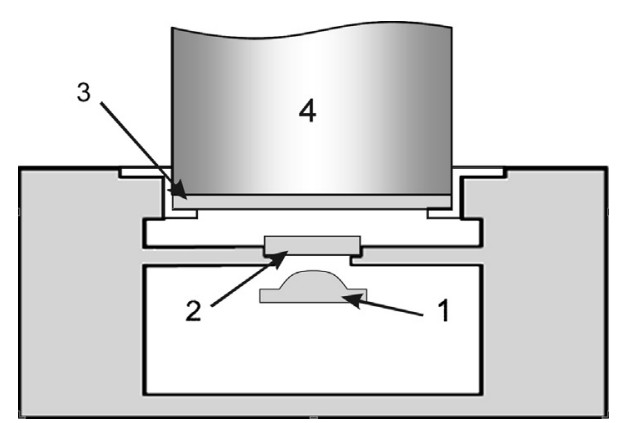

Fig. 1. Schematic diagram of the OSL unit: 1 - the stimulation source (blue or white LED); 2 - the sample; 3 - the separation filter; 4 - the photomultiplier tube. filter (3) of the UFS-2 type (spectral transmission range of 260$400 \mathrm{~nm})$ at a small distance $(1-2 \mathrm{~mm})$ from their surfaces. The light detector (4) was a photomultiplier tube of the FEU-142 type with reduced sensitivity to thermal radiation of a heater.

The filling of deep traps with charge carriers (electrons) is possible if the test samples are exposed to the UV radiation of a deuterium lamp at $670 \mathrm{~K}$ (Mil'man et al., 2008). This procedure is called thermal optical treatment (TOT). This radiation efficiently ionizes the F-centers at high temperatures and facilitates the filling of deep traps according to the following reactions:

$\mathrm{F}+\mathrm{h} v \rightarrow \mathrm{F}^{+}+\mathrm{e}^{-}$,

$\mathrm{e}^{-}+\mathrm{DT}_{i} \rightarrow \mathrm{e}_{\mathrm{DT}_{i}}^{-}$

where the $\mathrm{F}$ - and $\mathrm{F}^{+}$-centers are anion vacancies with two and one electrons, respectively; $\mathrm{h} \nu$ is the UV photon energy; $\mathrm{DT}_{i}$ is a deep $i$ trap $(i=1,2,3 \ldots)$; and $\mathrm{e}^{-}$and $\mathrm{e}_{\mathrm{DT}_{i}}^{-}$are a free electron and an electron captured in a deep i-trap, respectively. According to Eqs. (1) and (2), the filling of deep traps in the UV-irradiated samples was checked by the appearance of well-defined TL peak at $700 \mathrm{~K}$ $(\beta=2 \mathrm{~K} / \mathrm{s})$. It is significant that the intensity level of this hightemperature TL peak was undetectable when the samples were exposed to the UV irradiation at room temperature.

\section{Results and discussion}

If narrow-band sources of the stimulating light, e.g., blue and/or green LEDs, are used, the optimal ionization conditions are provided only for a comparatively small number of traps in their total distribution, and the ionization level is low. One of the methods proposed to improve the depletion efficiency of dosimetric traps and, consequently, the OSL registration efficiency is based on the optical stimulation source, the emission spectrum of which approaches the OSL stimulation spectrum most closely (Fig. 2, curve 1). These conditions are met by white LEDs of the KA1010PWC9AZC type (Kingbright), which deliver irradiance $E_{e}=4.1 \mathrm{~mW} / \mathrm{cm}^{2}$ at the sample. The emission spectrum is shown in Fig. 2 (curve 2). In addition, comparative studies use blue LEDs of the SDK-S469-5-10 type with $E_{\mathrm{e}}=3.5 \mathrm{~mW} / \mathrm{cm}^{2}$ and peak emission wavelength $\lambda_{\max }=470 \mathrm{~nm}$ (curve 3 ).

The OSL decay curves for a dosimetric signal stimulated by the radiation of white and blue LEDs are shown in Fig. 3a and b, respectively.

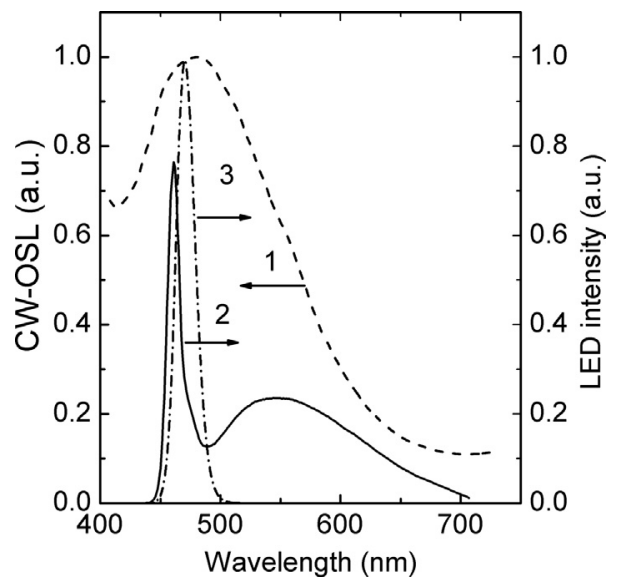

Fig. 2. The OSL stimulation spectrum of anion-defective $\alpha-\mathrm{Al}_{2} \mathrm{O}_{3}$ (curve 1 from BøtterJensen et al., 2003), emission spectra of the white LED (curve 2), and the blue LED (curve 3). 
a

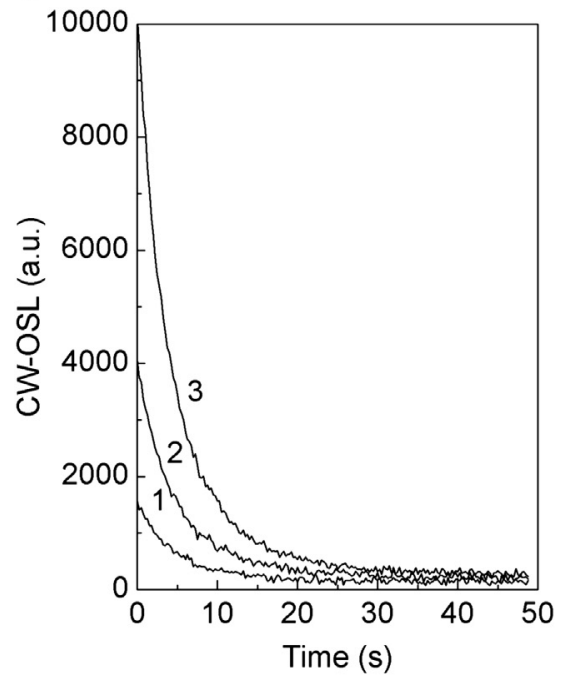

b

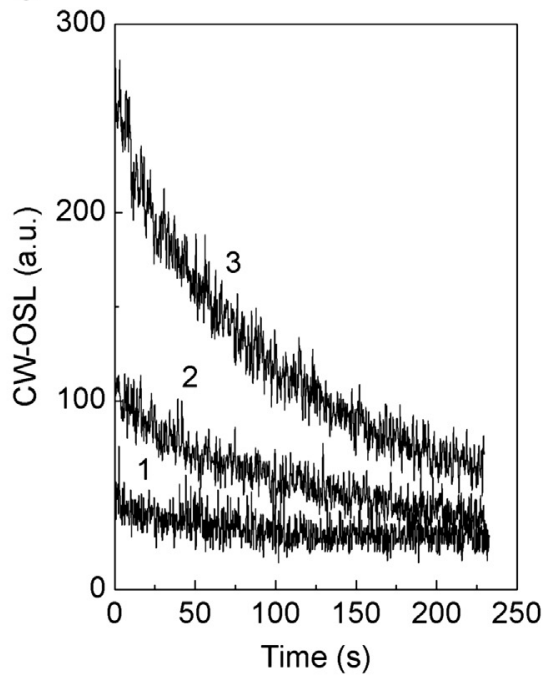

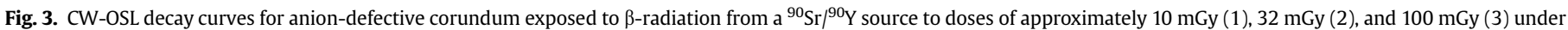
stimulation by white (a) and blue (b) LEDs.

These curves can be well described by the exponential dependence according to the following equation:

$I(t)=\sum_{k=1}^{n} I_{k} \cdot e^{-t / \tau_{k}}+I_{o}$

where $I_{k}$ and $\tau_{k}$ are the prefactor and the decay time constant of the $k$-component, respectively; $n$ is the number of exponential components in the analysis; $I_{0}$ is the constant component or background; and $t$ is the time of optical stimulation. To characterize the OSL yield, the values of the initial intensity $I_{i}$ and light sum $S$ are used, which are described by

$I_{i}=\sum_{k=1}^{2} I_{k}-I_{0}$,

$S=\int_{0}^{t_{1}}\left[I(t)-I_{0}\right] \mathrm{d} t$,

where $t_{1}$ is the measuring time. The value of $t_{1}$ is chosen to provide the decay of $I(t)$ down to the background level. Using Eqs. (3) and (5) can be reduced to

$S=\sum_{k=1}^{2} I_{k} \cdot \tau_{k}$,

by taking into account that the measuring time is $t_{1}>>\tau_{k}$.

Table 1

Parameters of the OSL decay curves as a function of the irradiation dose upon stimulation with the white LED.

\begin{tabular}{llll}
\hline Parameters of the OSL curves & \multicolumn{2}{l}{ Dose $(\mathrm{mGy})$} \\
\cline { 2 - 4 } & 10 & 32 & 100 \\
\hline$\tau_{1}(\mathrm{~s})$ & $4.8 \pm 0.5$ & $4.8 \pm 0.5$ & $4.5 \pm 0.5$ \\
$I_{i} \approx I_{1}$ (a.u.) & 1365 & 3740 & 10,305 \\
$I_{0}$ & 200 & 220 & 245 \\
$S \approx \tau_{1} I_{1}$ (a.u.) & $0.7 \cdot 10^{4}$ & $1.8 \cdot 10^{4}$ & $4.6 \cdot 10^{4}$ \\
\hline
\end{tabular}

The CW-OSL curves, shown in Fig. 3, can be expanded with sufficient accuracy using one exponent $(n=1)$. Tables 1 and 2 demonstrate the expansion results of the OSL curves obtained under white and blue LED stimulation, respectively.

According to the analysis of Tables 1 and 2, under the white LED stimulation, the main contribution to the OSL light sum is provided by rather fast component with a time constant of $\tau_{1} \approx 4.7 \mathrm{~s}$. In contrast, stimulation with the blue LED leads to a significant increase of the time constant up to $\sim 93 \mathrm{~s}$ and a decrease of the light sum by no less than a factor of two. From practical viewpoint the following results are of prime importance. As distinct from the blue LED, the use of white LED and the chosen geometry allows increasing the initial intensity of the OSL signal by more than 40 times and reducing the readout time of the stored light sum by no less than $\sim 20$ times (Fig. 2, Tables 1 and 2). This result is verified experimentally by absence of the "residual TL" after the OSL measurements under stimulation not only by blue LED but also by white LED.

OSL decay curves in Fig. 3 were obtained when the deep traps were not filled purposefully. However, their state might have a considerable effect on the behavior of the OSL curves. Thus, the behavior of OSL and the TL was studied in terms of their dependence on the population of the deep electronic trap emptied at $700 \mathrm{~K}$. The population level was controlled by a TOT time $\left(t_{\mathrm{TOT}}\right)$ over the range of $0-300 \mathrm{~s}$.

Fig. 4 shows the TL curves within the $300-800 \mathrm{~K}$ range before (curve 1) and after TOT at $670 \mathrm{~K}$ (curves 2, 3, 4, and 5). For the sample not subject to TOT, the TL curve has a dominant peak at $450 \mathrm{~K}$, which is called the main or dosimetric peak (curve 1 ). Due to TOT, the intensity of the main $450 \mathrm{~K}$ peak grows significantly (by

Table 2

Parameters of the OSL decay curves as a function of the irradiation dose upon stimulation with the blue LED.

\begin{tabular}{llll}
\hline Parameters of the OSL curves & \multicolumn{3}{l}{ Dose $(\mathrm{mGy})$} \\
\cline { 2 - 4 } & 10 & 32 & 100 \\
\hline$\tau_{1}$ (s) & $93 \pm 20$ & $92 \pm 9$ & $94 \pm 3$ \\
$I_{\mathrm{i}} \approx I_{1}$ (a.u.) & 25 & 79 & 252 \\
$I_{0}$ & 25 & 24 & 27 \\
$S \approx \tau_{1} I_{1}$ (a.u.) & $0.2 \cdot 10^{4}$ & $0.7 \cdot 10^{4}$ & $2.4 \cdot 10^{4}$ \\
\hline
\end{tabular}




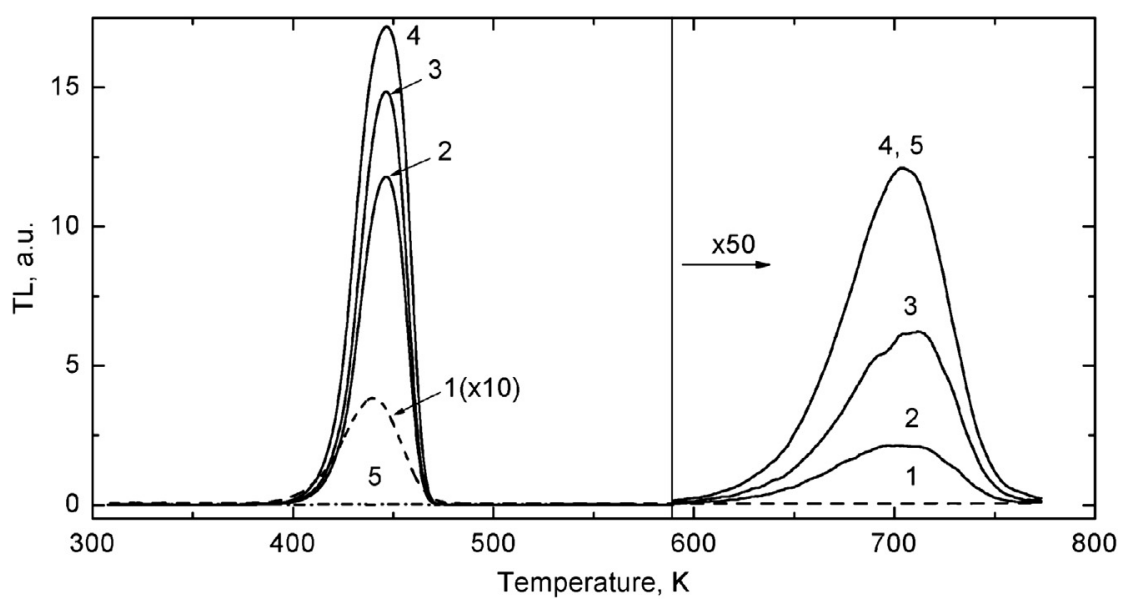

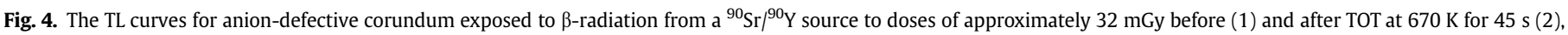
$120 \mathrm{~s}$ (3), and $300 \mathrm{~s}$ (4). The TL curve (5) is measured after TOT for $300 \mathrm{~s}$ without $\beta$-radiation.

a

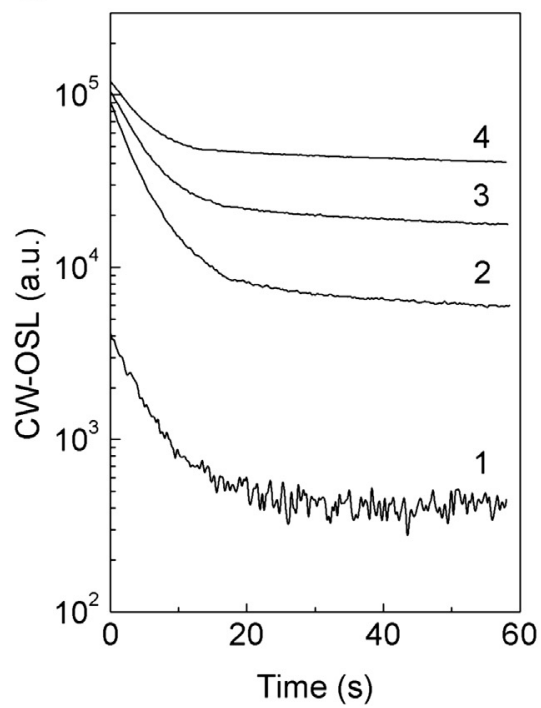

b

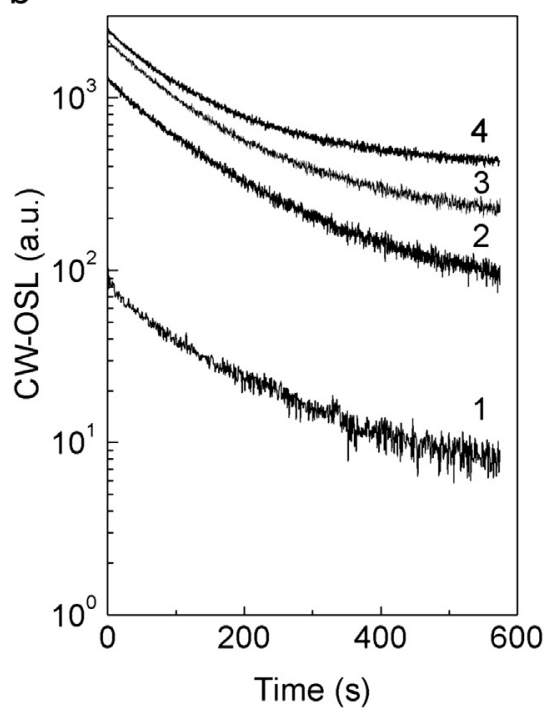

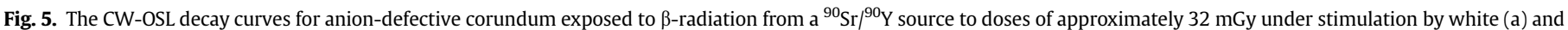
blue LEDs (b) before (1) and after TOT at $570 \mathrm{~K}$ for $45 \mathrm{~s}$ (2), $120 \mathrm{~s}$ (3), and $300 \mathrm{~s}$ (4).

more than an order of magnitude for $t_{\mathrm{TOT}}=300 \mathrm{~s}$ ), and a hightemperature peak appears at $700 \mathrm{~K}$ (curves 2-4). It should be noted that the TL curve (curve 5) measured for $300 \mathrm{~s}$ after TOT without $\beta$-irradiation is characterized by the $700 \mathrm{~K}$ peak, the intensity of which does not differ from that for the peak measured after $\beta$-irradiation with a test dose of $32 \mathrm{mGy}$.

As seen in Fig. 4, for small $t_{\mathrm{TOT}}$, the intensity of the $700 \mathrm{~K}$ peak is 2-3 orders of magnitude lower than the intensity of the dosimetric peak. The intensity of the aforementioned peaks increases with increasing $t_{\text {TOT }}$. The growth rate of the high-temperature $700 \mathrm{~K}$ peak, however, is considerably higher than that of the $450 \mathrm{~K}$ peak. This regularity can be supported by the decrease in the ratio of the 450 and $700 \mathrm{~K}$ peak intensities $\left(I_{450} / I_{700}\right)$ from $\geq 10^{5}$ (the dynamic range of the registration tract) to $\sim 70$ upon the change of $t_{\mathrm{TOT}}$ from 0 to $300 \mathrm{~s}$. The growth of peak intensity for 450 and $700 \mathrm{~K}$ dramatically slows down with increasing $t_{\mathrm{TOT}}$ up to $600 \mathrm{~s}$, resulting in saturation.

White and blue LEDs were used to observe the qualitative differences between the OSL curves for the samples with different populations of deep traps.
Fig. 5 presents the OSL curves for one sample exposed to $\beta$-radiation from a ${ }^{90} \mathrm{Sr} /{ }^{90} \mathrm{Y}$ source to a test dose of $32 \mathrm{mGy}$, with deep traps initially purposefully unfilled (curve 1 ) and then filled to different levels (curves 2, 3, and 4).

Table 3

Parameters of the OSL decay curves as a function of TOT time upon white LED stimulation.

\begin{tabular}{llll}
\hline Parameters of the OSL curves & \multicolumn{3}{l}{ TOT time $(\mathrm{s})$} \\
\cline { 2 - 4 } & 45 & 120 & 300 \\
\hline$\tau_{1}$ (s) & 4.7 & 6.6 & 9.7 \\
$I_{1}$ (a.u.) & $0.86 \cdot 10^{5}$ & $1.02 \cdot 10^{5}$ & $1.16 \cdot 10^{5}$ \\
$\tau_{1} I_{1}$ (a.u.) & $0.41 \cdot 10^{6}$ & $0.67 \cdot 10^{6}$ & $1.1 \cdot 10^{6}$ \\
$\tau_{2}$ (s) & 191 & 294 & 331 \\
$I_{2}$ (a.u.) & $0.05 \cdot 10^{5}$ & $0.17 \cdot 10^{5}$ & $0.43 \cdot 10^{5}$ \\
$\tau_{2} I_{2}$ (a.u.) & $0.93 \cdot 10^{7}$ & $0.49 \cdot 10^{7}$ & $1.4 \cdot 10^{7}$ \\
$I_{\mathrm{i}} \approx I_{1}+I_{2}$ & $0.91 \cdot 10^{5}$ & $1.19 \cdot 10^{5}$ & $1.59 \cdot 10^{5}$ \\
$S \approx \tau_{1} I_{1}+\tau_{2} I_{2}$ & $0.14 \cdot 10^{7}$ & $0.57 \cdot 10^{7}$ & $1.51 \cdot 10^{7}$ \\
$\tau_{1} I_{1} /\left(\tau_{2} I_{2}\right)$ & 0.44 & 0.14 & 0.08 \\
\hline
\end{tabular}


Table 4

Parameters of the OSL decay curves as a function of TOT time upon blue LED stimulation

\begin{tabular}{llll}
\hline Parameters of the OSL curves & \multicolumn{3}{l}{ TOT time $(\mathrm{s})$} \\
\cline { 2 - 4 } & 45 & 120 & 300 \\
\hline$\tau_{1}$ (s) & 92 & 95 & 93 \\
$I_{1}$ (a.u.) & 911 & 1654 & 1801 \\
$\tau_{1} I_{1}$ (a.u.) & $0.8 \cdot 10^{5}$ & $1.6 \cdot 10^{5}$ & $1.7 \cdot 10^{5}$ \\
$\tau_{2}$ (s) & 388 & 706 & 1262 \\
$I_{2}$ (a.u.) & 350 & 468 & 644 \\
$\tau_{2} I_{2}$ (a.u.) & $1.4 \cdot 10^{5}$ & $3.3 \cdot 10^{5}$ & $8.1 \cdot 10^{5}$ \\
$I_{\mathrm{i}} \approx I_{1}+I_{2}$ & 1261 & 2122 & 2445 \\
$S \approx \tau_{1} I_{1}+\tau_{2} I_{2}$ & $2.2 \cdot 10^{5}$ & $4.9 \cdot 10^{5}$ & $9.8 \cdot 10^{5}$ \\
$\tau_{1} I_{1} /\left(\tau_{2} I_{2}\right)$ & 0.62 & 0.48 & 0.21 \\
\hline
\end{tabular}

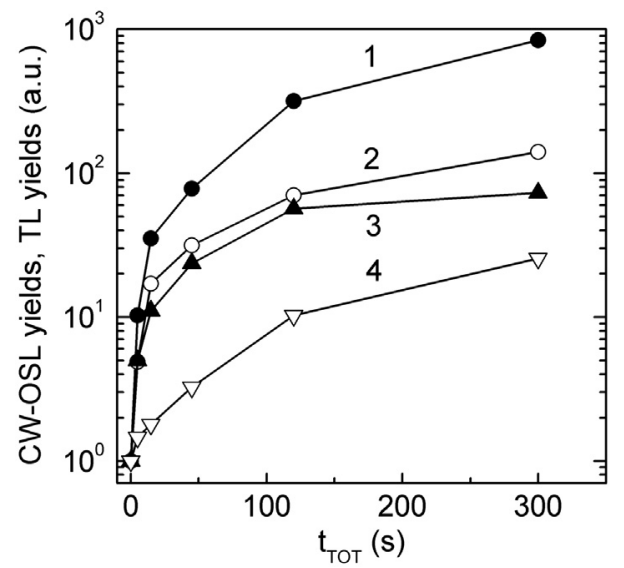

Fig. 6. Normalized dependences of the CW-OSL light sums under stimulation by white (1) and blue (2) LEDs and of the TL yield for the main dosimetric peak at $450 \mathrm{~K}$ (3) and the high temperature peak at $700 \mathrm{~K}(4)$ as a function of the TOT time at $670 \mathrm{~K}$.

The obtained data show (Fig. 5) that the OSL yield increases significantly with increasing TOT time. Simultaneously, the photoconversion $\mathrm{F} \rightarrow \mathrm{F}^{+}$occurs according to Eqs. (1) and (2), and the filling extent of the deep trap increases, as shown in Fig. 4. The CWOSL curves also become more complicated under stimulation due to TOT with both white and blue LEDs. Along with relatively fast decay components peculiar to the samples not subject to TOT (Fig. 5: (a) curve 1 and (b) curve 1), slower components appear (curves 2-4). Tables 3 and 4 summarize the expansion results for the CW-OSL curves of Fig. 5 according to Eqs. (3)-(6).

According to Fig. 5 and Tables 3 and 4, in the case of white LED stimulation, the time constants of the fast and slow components increase with increasing $\mathrm{t}_{\mathrm{TO}}$; in the case of blue LED stimulation, the constants of only the slow components increase. In addition, the data analysis reveals that the slow decay component increases both for blue and white LED stimulation with increasing $t_{\text {TOT }}$ contribution, which is confirmed by the reduction of the ratio $\tau_{1} I_{1} /$ $\left(\tau_{2} I_{2}\right)$ (Tables 3 and 4). Experimentally, increasing $t_{\mathrm{TOT}}$ above $300 \mathrm{~s}$ does not change the CW-OSL curves measured under stimulation by white and blue LEDs.

Fig. 6 summarizes the OSL and TL data obtained for aniondefective corundum exposed to TOT. From these data, it follows that the OSL sums under stimulation by white (curve 1 ) and blue (curve 2) LEDs and the TL yield within the range of the main dosimetric peak at $450 \mathrm{~K}$ (curve 3 ) correlate with the filling extent of the deep trap (curve 4). As a result of TOT, these yields increase no less than 50 times, and one can see a tendency to their saturation for a TOT exposition exceeding $200 \mathrm{~s}$. At the same time, as shown in Fig. 5, the contribution of the slow components to the OSL kinetics increases greatly.

Thus, the above results confirm the assumption about the influence of the different extents of the occupation of deep traps on the kinetic processes under optical stimulation. The complicated behavior of the OSL curves for the sample with filled deep traps indicates that the process involves several mechanisms. The discovered process of the OSL buildup may thus be due to two competing processes: the depletion of the main trap and its simultaneous filling through the phototransfer from deep traps.

\section{Conclusion}

1. Comparative studies of the OSL of anion-defective corundum exposed to white and blue LEDs were performed. It is shown that the use of a white LED combined with the chosen experimental geometry essentially increases the initial intensity of the OSL signal and its light sum and that it essentially reduces the readout time of dosimetric information compared to the use of a blue LED.

2. The influence of the filling extent of deep traps on the behavior of the OSL curves of the anion-defective corundum was studied. It is established that the filling of deep traps results in an increase of the OSL signal, which is an essential change in the shape of the OSL curve and the time parameters of decay. However, in this case, the detailed redistribution mechanism of carriers between traps of different deepness requires further study.

\section{Acknowledgments}

This work was supported by Presidium of the Ural Branch of the Russian Academy of Sciences (project no. 12-U-2-1032) and by the Russian Foundation for Basic Research (project no. 12-08-31186).

\section{References}

Bøtter-Jensen, L., McKeever, S.W.S., Wintle, A.G., 2003. Optically Stimulated Luminescence Dosimetry. Elsevier Science, p. 355.

Denis, G., Akselrod, M.S., Yukihara, E.G., 2011. J. Appl. Phys. 109 (10), 10, 104906.

Hashimoto, T., Nakagawa, T., Hong, D.-G., Takano, M., 2002. J. Nucl. Sci. Technol. 39 (1), 108-109.

McKeever, S.W.S., Akselerod, M., 1999. Radiat. Protec. Dosimetry. 84, 317-320.

Miller S.D., Smith L.E., Skorpic J.R., 2006. Apparatus and methods for OSL-based, remote radiation monitoring and spectrometry. USA Patent 7009181, 03.07.

Mil'man, I.I., Moiseykin, E.V., Nikiforov, S.V., Solov'ev, S.V., Revkov, I.G., Litovchenko, E.N., 2008. Phys. Solid State 50 (11), 2076-2080.

Solov'ev, S.V., Milman, I.I., Syurdo, A.I., 2012. Phys. Solid State 54 (4), 726-734.

Springis, M., Kulis, P., Veispas, A., Tale, I., 1995. Radiat. Meas. 24, 453-456.

Surdo, A.I., Kortov, V.S., 2004. Radiat. Meas. 38, 667-671.

Surdo, A.I., Milman, I.I., Vlasov, M.I., Il'ves, V.G., Sokovnin, S.Yu., 2013. Tech. Phys. Lett. 39 (1), 5-8.

Walker, F.D., Colyott, L.E., Larsen, N.A., McKeever, S.W.S., 1996. Radiat. Meas. 26 711-718.

Yukihara, E.G., McKeever, S.W.S., 2011. Optically Stimulated Luminescence: Fundamentals and Applications. John Wiley \& Sons, p. 362. 